\title{
OPTIMAL CHARACTERIZATION OF LF MAGNETIC FIELD USING MULTIPOLES
}

\author{
R. Scorretti, R. Takahashi ${ }^{(*)}$, L. Nicolas and N. Burais \\ CEGELY UMR 5005 - Ecole Centrale de Lyon \\ 36 av. Guy de Collongues - 69134 Ecully (France) \\ ${ }^{(*)}$ Universidade Federal de Minas Gerais (UFMG) \\ Belo Horizonte (Brasil) \\ e-mail : riccardo.scorretti@ec-lyon.fr
}

\begin{abstract}
The LF magnetic field (50 Hz- $100 \mathrm{kHz}$ ) generated in the air by electrical appliances is characterised using multipoles. The maximum likelihood estimation of an equivalent multipolar source is computed using a genetic algorithm. The choice of the position and the number of measurement points are discussed.
\end{abstract}

\section{INTRODUCTION}

The increasing number of electrical appliances in the daily life, rises the question of the interaction of these fields with the living systems. The effects on the human health of occasional (unwanted) exposure to the electromagnetic fields are still not well known. For this reason, the governments have imposed some restrictions on the fields generated in the air by electrical appliances. In the case of low frequency $(50 \mathrm{~Hz}-100 \mathrm{kHz})$ magnetic fields, these limits are defined on the basis of the induced currents into the human body. Unfortunately, these currents are extremely weak, and a direct measurement is impossible. The simplest model to compute these current is a 1D model [1], where the magnetic field is supposed to be uniform. Clearly, this is not a realistic situation in proximity of the appliance, and by consequence the reference levels on the field which derive from this model are too restrictive $[2,3]$. Thus it is important to be able to characterize the fields generated in the air by electrical appliances using experimental data. The main idea is to perform some measurements of the magnetic field in the air, and use them to fit the parameters of an equivalent source. This source can replace the real appliance, in the computation of the induced currents in the human body. The simplest model of equivalent source which has been proposed is a magnetic dipole $[4,5]$. However, a simple dipole may be not representative enough of the field distribution, depending upon the kind of the appliance [6], and on the distance from it. In this case, a multipolar source can be used. Compared to the magnetostatic case, the main difficulty with this technique in the harmonic regime is the need to measure both the amplitude and the phase [7] of the magnetic field. However, the measurement of the phase is really necessary only when dealing with rotating fields. This paper presents an approach based on the maximum likelihood estimation (MLE) of a multipolar source, using a genetic algorithm (GA). First, the formulation of the problem is presented. Then, the method is tested on a simple source (a coil) using computer-generated data. The choice of the measurement points is discussed. Finally, the application to a real device is presented.

\section{FORMULATION OF THE PROBLEM}

The simplified Maxwell equations for the harmonic, quasimagnetostatic case [8] are :

$$
\begin{aligned}
\vec{\nabla} \cdot \vec{B} & =0 \\
\vec{\nabla} \times \vec{H} & =\vec{J}
\end{aligned}
$$

The air surrounding the electrical appliance is supposed to be free from currents $(\vec{J}=0)$, so that the magnetic field $\vec{H}$ can be described using either a scalar potential $\psi$, or a vector potential $\vec{A}$ :

$$
\vec{H}=-\vec{\nabla} \psi=\frac{1}{\mu_{o}} \vec{\nabla} \times \vec{A}
$$

Both these potentials obeys to the Laplace's equation, and thus can be described by a multipolar development [9] :

$$
\begin{gathered}
\psi=\sum_{n=1}^{\infty}\left(\frac{R}{r}\right)^{n+1} \sum_{m=-n}^{n} \underline{A}_{n, m} Y_{n}^{m}(\vartheta, \varphi) \\
\vec{A}=-\sum_{n=1}^{\infty} \frac{1}{n}\left(\frac{R}{r}\right)^{n+1} \underline{A}_{n, m} \vec{r} \times \vec{\nabla} Y_{n}^{m}(\vartheta, \varphi)
\end{gathered}
$$

where $\underline{A}_{l, m}$ are the coefficients of the development, $R$ is an arbitrary distance, and $Y_{n}^{m}(\vartheta, \varphi)$ are the spherical harmonics [10]. The coefficients $\underline{A}_{n, m}$ depend upon the choice of $R$ and the coordinate system (i.e. the position and the orientation of the equivalent source).

\section{a. Choice of $R$}

Eq. 4 and 5 hold outside the sphere of radius $R$. Thus $R$ must be large enough, such that the appliance is contained inside this sphere. On the other hand, to achieve an accurate characterisation of the field in the, $R$ should be chosen as 
small as possible. However, the choice of $R$ is not crucial, in that a different choice $R^{\prime} \neq R$ leads to a simple scaling of the coefficients.

\section{b. Choice of the coordinate system}

The choice of the coordinate system (i.e. the centre and the orientation of the multipole) turns out to be very important for the quality of the characterisation. First, it should be observed that the number $P$ of the coefficients grows quickly with the order $N$ of the development :

Table 1: number of coefficients of the development
\begin{tabular}{|r|c|c|c|}
\hline order & $\mathrm{N}=1$ & $\mathrm{~N}=2$ & $\mathrm{~N}=3$ \\
\hline coefficients & $\mathrm{P}=3$ & $\mathrm{P}=8$ & $\mathrm{P}=15$ \\
\hline
\end{tabular}

From the mathematical point of view, the problem is definitely solved by the eq. 4 or 5 . However, in practical applications the infinite sums in eq. 4 and 5 have to be bounded to a certain order (i.e. $n \leq N$ ). It turns out that a smart choice of the coordinate system improves the accuracy of the fit, and avoids the unnecessary complications dues to high-order harmonics. An explication of this fact is that the symmetries of the system may be (implicitly) taken into account in the choice of the coordinate system.

\section{c. Maximum Likelihood Estimation of the coefficients}

Assume now that $R$ and the coordinate system have been chosen, and that the magnetic field $\vec{H}$ is measured onto a set of $M$ points. For sake of simplicity, assume that the field is periodic with time, and that only the fundamental component ${ }^{(1)}$ is considered : thus a $3 \mathrm{D}$ complex vector $\vec{H}$ is associated to each measurement point. It can be observed that the field depends linearly upon the coefficients :

$$
[\underline{\mathbf{H}}]=[\mathbf{C}] \cdot[\underline{\mathbf{A}}]+\sigma^{2}[\mathbf{I}]
$$

where the term $\sigma^{2}[\mathbf{I}]$ models a white gaussian measurement noise, and the coefficients and the measured values of the field are arranged into the vectors $[\underline{\mathbf{A}}]$ and $[\underline{\mathbf{H}}]$ respectively. The maximum likelihood estimation [11] of the coefficients [스] and its covariance matrix can be computed using the well known formulas :

$$
\begin{gathered}
\widehat{\widehat{\mathbf{A}}]}=\left([\mathbf{C}]^{H}[\mathbf{C}]\right)^{-1}[\mathbf{C}]^{H} \cdot[\underline{\mathbf{H}}] \\
\operatorname{cov}[\underline{\widehat{\mathbf{A}}}]=\frac{1}{3 M-P}\left([\mathbf{C}]^{H}[\mathbf{C}]\right)^{-1} \cdot \\
\cdot[\underline{\mathbf{H}}]^{H}\left([\mathbf{I}]-[\mathbf{C}]\left([\mathbf{C}]^{H}[\mathbf{C}]\right)^{-1}[\mathbf{C}]^{H}\right)[\underline{\mathbf{H}}]
\end{gathered}
$$

(1) Actually, this can be assumed without loss of generality. In fact the Laplace's equation is linear: thus each component can be considered independently from the others, by the superposition principle.
The number of measurement points $M$ must be large enough, for the system in eq. 7 to be over determined : $3 M>P$.

\section{d. The optimisation problem}

The "core" of the method (fig. 1) consists in choosing the coordinate system in an somehow optimal way. To this purpose, a genetic algorithm $[12,13]$ is used. The parameters to be optimised are the coordinates of the centre of the source : $x_{o}, y_{o}, z_{o}$. The cost function $j$, to be minimized, is the mean square reconstruction error :

$$
j=\frac{1}{M} \sum_{k=1}^{M}\left\|\vec{H}_{k}-\underline{\vec{H}}_{k}^{(M P)}\right\|^{2}
$$

where $\underline{\vec{H}}_{k}^{(M P)}$ is the magnetic field computed in the k-th measurement point, using the equivalent multipole.



Figure 1 : sketch of the method

\section{RESULTS}

First, the effectiveness of the method has been tested with a simple source (a coil). In this case, the "measurement" data have been computed using analytical formulas [8]. Then, the method has been applied on real measurements from a test device.

\section{a. Application to a circular coil}

The coil (radius $a=40 \mathrm{~cm}$, current $I=1 \mathrm{~A}$ ) is placed at the origin, the axis oriented along the $\mathrm{z}$-direction, and modelled with a third-order multipole. First, 36 measurements points (fig. 2) have been taken on a sphere of radius $1 \mathrm{~m}$. The coefficients has been first estimated when the multipole is placed at the origin (real position), at two other arbitrary positions (case a, b - see tab. 2), and at the position obtained with the genetic algorithm (G.A.) Only the position of the coordinate system has been optimised. 




Figure 2 : the coil, with the measurement points.

First of all, one sees that the optimised location computed by the genetic algorithm is very close to the real position :

Table 2 : center of the equivalent multipole
\begin{tabular}{|l|l|l|l|}
\hline real position & \multicolumn{1}{|c|}{ case $(\mathrm{a})$} & \multicolumn{1}{c|}{ case $(\mathrm{b})$} & G.A. \\
\hline \hline $\mathrm{x}_{\mathrm{o}}=0$ & $\mathrm{x}_{\mathrm{o}}=0.1$ & $\mathrm{x}_{\mathrm{o}}=0.3$ & $\mathrm{x}_{\mathrm{o}}=-0.0217$ \\
$\mathrm{y}_{\mathrm{o}}=0$ & $\mathrm{y}_{\mathrm{o}}=-0.05$ & $\mathrm{y}_{\mathrm{o}}=-0.3$ & $\mathrm{y}_{\mathrm{o}}=-0.0090$ \\
$\mathrm{z}_{\mathrm{o}}=0$ & $\mathrm{z}_{\mathrm{o}}=-.030$ & $\mathrm{z}_{\mathrm{o}}=0.4$ & $\mathrm{z}_{\mathrm{o}}=0.0014$ \\
\hline
\end{tabular}

In fig. 3 the absolute values of the coefficients have been plotted. One observe that when the location of the multipole is chosen arbitrarily (case a, b), some high-order harmonic terms appear. These terms have no physical meaning, and are introduced only to fit the data. Thus the solution obtained by the genetic algorithm is, in some way, the simplest one.

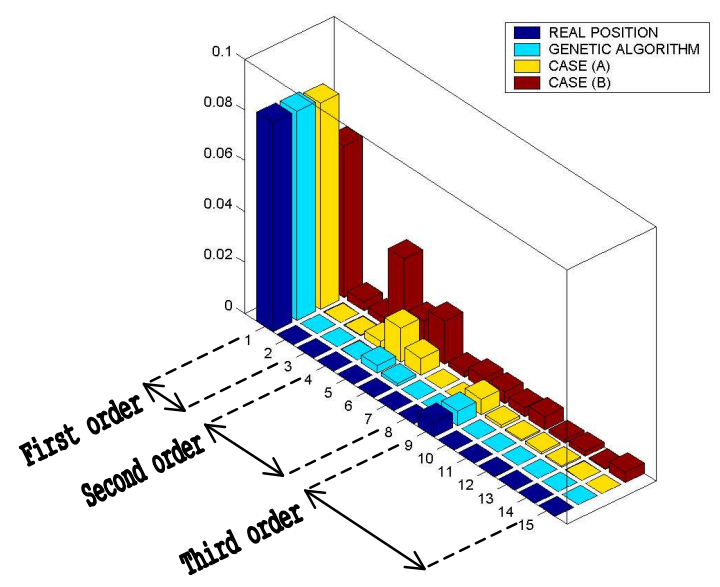

Figure 3 : coefficients of the multipole

In order to quantify the effectiveness of the solution, we define in each point the error criterion :

$$
\varepsilon=\left\|\underline{\vec{H}}-\underline{\vec{H}}^{(M P)}\right\| / \max \|\underline{H}\| \cdot 100 \%
$$

This criterion has been computed over a sphere of radius $2 \mathrm{~m}$, centred into the origin. The maximum values of $\varepsilon$ obtained are : $3.3 \%$ in the case (a), $44 \%$ in the case (b), and $0.87 \%$ when the centre is optimised. Thus, when the position of the multipole is optimised, a better agreement with the data can be achieved.

\section{b. The choice of the measurement points}

An important question which arises when dealing with a real appliance is where to perform the measurements of the field. In the previous section, the measurement points have been placed onto a sphere which surround the source of the field. This is seldom a realistic situation, in that one may actually not be able to locate the field sensors all around the appliance. Moreover, the effectiveness of the method has been validated a posteriori, using a second set of data. Even if this is an unavoidable step, it is important to have an a priori criterion about the goodness of the choice of the measurement points. To this purpose, some other configurations of the measurement points have been simulated. Among them, a case which has a high practical interest [4] is when the points are placed onto a straight line (fig.4). In this case the method fails. This result merits to be investigated more carefully.

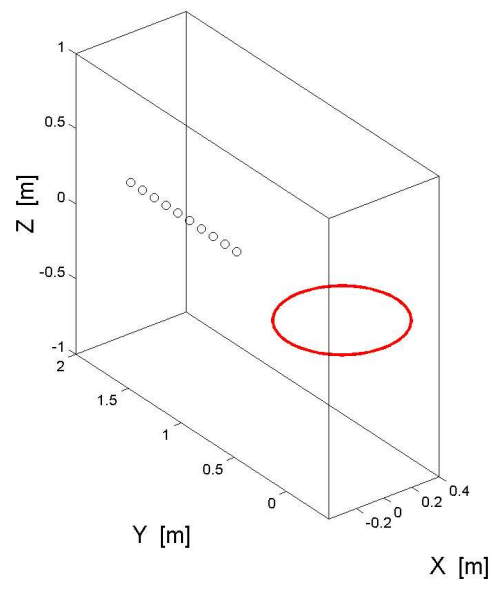

Figure 4 : the measurement points, placed onto a straight line.

First of all, it can be observed that the estimated values of the coefficients (fig. 5) are meaningless : not only the order of magnitude is wrong $\left(10^{5}\right.$ compared to $\left.10^{-1}\right)$, but the dipolar character of the coil cannot be argued in any way.

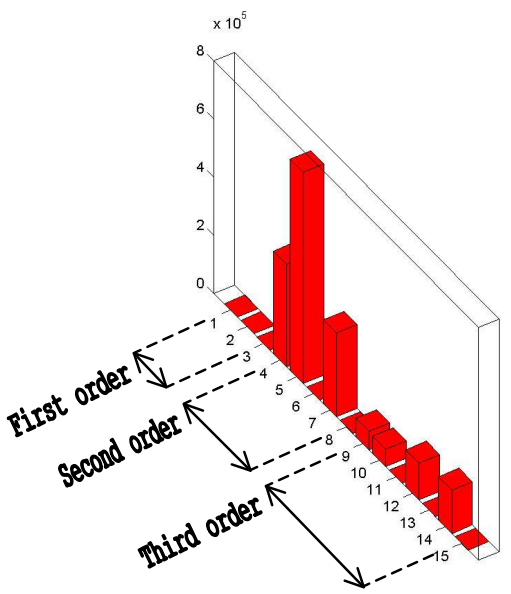

Figure 5 : estimated coefficients for the points placed onto a straight line 
Most importantly, we find that the multipole fits perfectly the field in the points used for the estimation ( $\varepsilon<10^{-5} \%$ ), but fails utterly elsewhere $\left(\varepsilon=10^{8} \%\right)$. We have checked that the problem is not the genetic algorithm : even if one imposes that the multipole is located in the origin, the result is not improved. Indeed, one sees that the problem is intrinsic to the choice of the measurement points. This is confirmed by the fact that the condition number of the matrix $[\mathbf{C}]$ in eq. 7 has been estimated to $3 \cdot 10^{32}$. On the other hand, one may think to make use of the condition number of [C] to find out some useful a priori informations about the goodness of the choice of the measurement points.



Figure 6 : the test-device (right) and the flux density computed using a 3D Finite Elements model (left)

\section{c. Application to a real test-appliance.}

The method has been tested on the device in fig. 6 , which is composed of a magnetic core with two air-gap, excited by a coil. Two set of measurements have been taken :

a) on a plane above the device (64 points)

b) on a straight line (15 points)

The set (a) has been used to identify a multipole of the first and of the third order. The set (b) is used to validate these models. In both cases, a good localization of the device is found. Moreover, the two models are compatibles : in fact the first-order terms of the two models are close, and dominate the high-order terms. The error criterion are :

Table 3 : error criterion for the models of the first and third order

\begin{tabular}{|r||l|l|}
\hline first order & set (a) $: \varepsilon<16 \%$ & set (b) : $\varepsilon<22 \%$ \\
\hline third order & $\operatorname{set}(\mathrm{a}): \varepsilon<3 \%$ & $\operatorname{set}(\mathrm{b}): \varepsilon<64 \%$ \\
\hline
\end{tabular}

The first-order model is more representative than the thirdorder one. One observes that for the third-order model, the condition number of $[\mathbf{C}]$ is about $10^{3}$ : thus a better choice of the measurement points is likely to improve the results.

\section{CONCLUSIONS}

A method to model the LF magnetic field generated by electrical appliances by an equivalent multipole is studied. The choice of the measurement points appears to be crucial : it would be interesting to explore the possibility of improving the estimation using multi-objective formulations.

\section{REFERENCES}

[1] ICNIRP, "Guidelines for limiting exposure to timevarying electric, magnetic, and electromagnetic fields (up to $300 \mathrm{GHz}) "$, Health Phys., vol. 74, no 4 (1998).

[2] N. Burais, P. Baraton, J.Y. Gaspard, "Modélisation numérique des courants induits dans le corps humain par des dispositifs électromagnétiques dans la gamme des fréquences

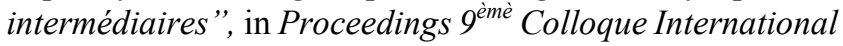
sur la Compatibilité Electromagnétique (1998).

[3] J.Y. Gaspard, N. Burais, F. Deschamps, "Exposition des personnes aux champs électromagnétiques : des nouvelles contraintes pour l'industrie électrique", in Proceedings $10^{\text {èmè }}$ Colloque International sur la Compatibilité Electromagnétique (2000).

[4] L. Zaffanella, T. Sullivan, I. Visintainer, "Magnetic field characterization of electrical appliances as point source through in situ measurements", IEEE Trans. Pow. Del., vol. 12, no. 1 (1997).

[5] K. Yamazaki, T. Kawamoto, "Simple estimation of equivalent magnetic dipole moment to characterize ELF magnetic fields generated by electric appliances incorporating harmonics", IEEE Trans. EMC, vol. 43, no. 2 (2001).

[6] S. Salomon, O-Mun Kwon, "External fields of permanent magnet motors", in Proceeding $13^{\text {th }}$ Compumag (2001), vol. 3 , pp. 58,59 .

[7] C.E. Lyon, "Modelling of magnetic fields generated by low frequency sources using multipole techniques", $\mathrm{PhD}$ thesis (1994), Washington State University, USA, pp. 8-11

[8] E. Durand, Magnétistatique, Masson, Paris (1968).

[9] J. Lorange, Couplage des inductances par rayonnement magnétique. Etude théorique et expérimentale, $\mathrm{PhD}$ thesis (2001), Laboratoire d'Electrotechnique de Grenoble (LEG), France, pp. 11-30

[10] www . mathworld.wolfram.com/SphericalHa rmonic.html

[11] G. Strang, Introduction to Applied Mathematics, Wellesley, Cambridge (1986), p. 398.

[12] D.E. Goldberg, Genetic Algorithms in search, optimization and machine learning, Addison-Wesley, Reading, MA (1989).

[13] R. Takahashi, J.A. Vasconcelos, J.A. Ramirez, L. Krahenbuhl, "A multiobjective lethodology for evaluating genetic operators”, IEEE Trans. Mag. vol. 39, no. 3 (2003) 